Original Article

\title{
2D AND 3D-QSAR ANALYSIS OF AMINO (3-((3, 5-DIFLUOR0-4-METHYL-6- PHENOXYPYRIDINE-2-YL) OXY) PHENYL) METHANIMINIUM DERIVATIVES AS FACTOR XA INHIBITOR
}

\author{
SMITA SUHANE ${ }^{1}$, A. G. NERKAR ${ }^{2}$, KUMUD MODI ${ }^{3}$, SANJAY D. SAWANT ${ }^{4}$
}

1Department of Applied Science, Dr. D. Y. Patil Institute of Technology, Pimpri, Pune 411018 India, ${ }^{2}$ Department of Pharmaceutical Chemistry, R. C. Patel Institute of Pharmaceutical Education and Research, Karwand Naka, Shirpur 425405 India, ${ }^{3}$ P. M. B Gujarati Science College, 1, Nasia Road, Indore 452001 India, ${ }^{4}$ Department of Pharmaceutical Chemistry, Smt. Kashibai Navale College of Pharmacy, Kondhwa-Saswad Road, Kondhwa (Bk.), Pune 411048

Email: smita3472@gmail.com

Received: 30 Jun 2017 Revised and Accepted: 08 Jan 2019

\section{ABSTRACT}

Objective: The main objective of the present study was to evolve a novel pharmacophore of methaniminium derivatives as factor Xa inhibitors by developing best 2D and 3D QSAR models.

The models were developed for amino (3-((3, 5-difluoro-4-methyl-6-phenoxypyridine-2-yl) oxy) phenyl) methaniminium derivatives as factor Xa inhibitors.

Methods: With the help of Marvin application, 2D structures of thirty compounds of methaniminium derivatives were drawn and consequently converted to 3D structures. 2D QSAR using multiple linear regression (MLR) analysis and PLS regression method was performed with the help of molecular design suite VLife MDS 4.3.3. 3D QSAR analysis was carried out using k-Nearest Neighbour Molecular Field Analysis (k-NN-MFA).

Results: The most significant 2D models of methaniminium derivatives calculated squared correlation coefficient value 0.8002 using multiple linear regression (MLR) analysis. Partial Least Square (PLS) regression method was also employed. The results of both the methods were compared. In 2D QSAR model, T_C_O_5, T_2_O_2, s log p, T_2_O_1 and T_2_O_6 descriptors were found significant.

The best 3D QSAR model with k-Nearest Neighbour Molecular Field Analysis have predicted $\mathrm{q}^{2}$ value 0.8790 , q2_se value 0.0794 , pred $\mathrm{r}^{2}$ value 0.9340 and pred_r ${ }^{2}$ se value 0.0540 . The stepwise regression method was employed for anticipating the inhibitory activity of this class of compound. The 3D model demonstrated that hydrophobic, electrostatic and steric descriptors exhibit a crucial role in determining the inhibitory activity of this class of compounds.

Conclusion: The developed 2D and 3D QSAR models have shown good $\mathrm{r}^{2}$ and $\mathrm{q}^{2}$ values of 0.8002 and 0.8790 respectively. There is high agreement in inhibitory properties of experimental and predicted values, which suggests that derived QSAR models have good predicting properties.

The contour plots of 3D QSAR (k-NN-MFA) method furnish additional information on the relationship between the structure of the compound and their inhibitory activities which can be employed to construct newer potent factor Xa inhibitors.

Keywords: QSAR, K-Nearest Neighbour Molecular Field Analysis, Amino (3-((3, 5-difluoro-4-methyl-6-phenoxypyridine-2-yl) oxy) phenyl) methaniminium derivatives, Factor Xa

(C) 2019 The Authors. Published by Innovare Academic Sciences Pvt Ltd. This is an open access article under the CC BY license (http://creativecommons.org/licenses/by/4.0/] DOI: http://dx.doi.org/10.22159/ijpps.2019v11i2.21067

\section{INTRODUCTION}

The structure of any molecule dictates its properties. By modifying the chemical structure of any compound, its biological activities also get changed. In other words, the biological activity of a compound is a function of its chemical structure. QSAR suggests that if a group of chemicals show the same mechanism of action towards a target then alteration in the biological activity also alters chemical, structural and physical properties [1].

QSAR methods are not only used in drug designing but are widely used in other sciences too, i.e. in biology, toxicology [2-3] environmental toxicology [4], agrochemistry, pharmaceutical chemistry etc. The QSAR is also used to determine the initial and final point of synthesis [5]. This, in turn, reduces the number of compounds that could be practically/experimentally synthesized. This part of QSAR is not only beneficial for the pharmaceutical industry but also to environmental regulatory authority and human beings for the reduction in toxic effects [6-10].

QSAR models are not only used for prediction of properties but are also helpful in selection of alternative mechanism of action, determination of useful structural characteristics, projecting new design methodologies and help in proposing new hypotheses for future research work [11]. Thus, QSAR decreases the cost, time and human resources to make that drug reachable to the patient.

The injury to the blood vessel causes the body to use platelets and fibrin to make a blood clot or thrombus. The process of formation of the blood clot is called thrombosis or coagulation. When clotting takes place excessively and the thrombus or clot breaks and detaches from its side then an embolus is produced [12-13]. When thrombus occlude the blood vessel then the supply of oxygen to the tissue through blood is interrupted which causes cell death or necrosis.

The thrombi in the arteries are platelet rich with small fibrin requiring antiplatelet therapy primarily in ischemic heart disease. For venous thromboembolism and atrial thromboembolism having large fibrin burden, for example, deep vein thrombosis (DVT), pulmonary embolism (PE), stroke prevention in atrial fibrillation (AF) [14-16] and mechanical prosthetic heart valve, long term anticoagulation is necessary.

Venous arterial thromboembolism continues to be a major cause of morbidity and mortality globally. Research is going on for long term 
prevention of thromboembolic episode because of the large burden of other thrombotic and thromboembolic disorders.

Unfractionated heparin was the first anticoagulant (injectable) developed a century ago. Later on, heparin was further modified and refined resulting in the development of low molecular weight heparin and site-specific (injectable) fondaparinux (factor Xa indirect inhibitor).

Similarly, in the field of oral anticoagulants, classical vitamin K antagonists have developed around 70 y ago. Vitamin K antagonist acts by competitively inhibiting enzymes involved in the synthesis of factor II, VII, IX and X in the liver. Around thirty proteins are needed for coagulation cascade [17]. Vitamin $\mathrm{K}$ antagonist are having many limitations such as the need for INR monitoring due to the narrow therapeutic window, numerous drug and food interactions and increased chances of bleeding. Therefore, the need for site-specific oral anticoagulant having less chance of bleeding, less drug interaction and not requiring monitoring was sought and development of other antithrombotic agents started [18-23].

At present, heparin and oral anticoagulants are only available therapy for treating thrombotic disorders. Previously, benzamidine
[24] and arylamide compounds have been reported as factor Xa inhibitors [25] but still, oral anticoagulants which do not require monitoring and have less chances of bleeding are essayed to decrease the mortality and morbidity rate.

Factor Xa is present at the central position of coagulation cascade or at the converging point of the extrinsic and intrinsic pathway. The intrinsic and extrinsic pathway model splits up the coagulation process into two fragments. Consequently, Factor Xa has emerged as an attractive target for the development of antithrombotic drugs.

\section{MATERIALS AND METHODS}

Software which was employed to present study includes Marvin Sketch, Chem Bio Draw Ultra 12.0 and Vlife MDS 4.3.3 [26]. In the present study, a dissimilar set of amino (3-( (3, 5-difluoro-4methyl-6-phenoxypyridine-2-yl) oxy) phenyl) methaniminium derivatives were assessed as factor Xa inhibitor as antithrombotic agents. Set of 30 molecules was used as a dataset with their inhibitory activities towards factor Xa. The molecular structures of amino (3-( (3, 5-difluoro-4-methyl-6-phenoxypyridine-2-yl) oxy) phenyl) methaniminium derivatives with their binding affinity for factor Xa are presented in table 1.

Table 1: The structures of compound and Ki values for factor Xa<smiles>[R]c1ccc(Oc2nc(Oc3cccc(C(N)=[NH2+])c3)c(F)c(C)c2F)c([R])c1[R]</smiles>

\begin{tabular}{|c|c|c|c|c|}
\hline Compound & $\mathbf{R}^{\prime}$ & R" & $\mathbf{R}$ & Ki \\
\hline 3 & -1 & $\mathrm{H}$ & $\mathrm{H}$ & 5.920 \\
\hline 6 & & $\mathrm{H}$ & $\mathrm{H}$ & 5.886 \\
\hline 8 & & $\mathrm{H}$ & $\mathrm{H}$ & 5.869 \\
\hline 10 & & & $\mathrm{H}$ & 5.850 \\
\hline 13 & c & $\mathrm{H}$ & $\mathrm{H}$ & 5.769 \\
\hline 15 & & $\mathrm{H}$ & $\mathrm{H}$ & 5.744 \\
\hline 16 & & $\mathrm{H}$ & $\mathrm{H}$ & 5.744 \\
\hline 18 & & $\underset{1}{\mathrm{OH}}$ & $\mathrm{H}$ & 5.698 \\
\hline 21 & & $\mathrm{H}$ & $\mathrm{H}$ & 5.677 \\
\hline 23 & & $\mathrm{H}$ & $\mathrm{H}$ & 5.657 \\
\hline 26 & & $\mathrm{H}$ & & 5.619 \\
\hline 27 & & $\mathrm{H}$ & & 5.619 \\
\hline 32 & $F$ & $\mathrm{H}$ & $\mathrm{H}$ & 5.494 \\
\hline 33 & -1 & $\mathrm{H}$ & $\mathrm{H}$ & 5.481 \\
\hline 36 & & $\mathrm{H}$ & $\underset{l}{\mathrm{OH}}$ & 5.346 \\
\hline
\end{tabular}




\begin{tabular}{|c|c|c|c|c|}
\hline 39 & $\mathrm{O}_{\mathrm{N}} \mathrm{c}^{\prime}$ & $\mathrm{H}$ & $-\mathrm{CH}_{3}$ & 5.301 \\
\hline 40 & & $\mathrm{H}$ & $\mathrm{H}$ & 5.301 \\
\hline 41 & & $\mathrm{H}$ & $\mathrm{H}$ & 5.301 \\
\hline 42 & & $\mathrm{H}$ & $\begin{array}{ll}\mathrm{I} & \mathrm{O} \\
\mathrm{H}_{2} \mathrm{C}\end{array}$ & 5.300 \\
\hline 43 & & $\mathrm{H}$ & $\mathrm{H}$ & 5.301 \\
\hline 44 & & $\mathrm{H}$ & & 5.301 \\
\hline 45 & & $\mathrm{H}$ & & 5.301 \\
\hline 46 & & $\mathrm{OH}$ & $\mathrm{H}$ & 5.301 \\
\hline 47 & & $\mathrm{P}_{, \mathrm{H}}$ & $\mathrm{OH}_{1}$ & 5.301 \\
\hline 48 & & $\mathrm{H}$ & $\underset{\mathrm{OH}}{\mathrm{OH}}$ & 5.301 \\
\hline 49 & $\mathrm{OH}_{1}$ & $\mathrm{H}$ & $\mathrm{H}$ & 5.301 \\
\hline 50 & $\mathrm{H}$ & $\mathrm{H}$ & $\mathrm{H}$ & 5.301 \\
\hline 51 & & $\mathrm{H}$ & $\mathrm{OH}_{1}$ & 5.301 \\
\hline 52 & & $\mathrm{H}$ & $\underset{\mathrm{OH}}{\mathrm{OH}}$ & 5.301 \\
\hline 53 & & $\mathrm{H}$ & $\mathrm{H}$ & 5.283 \\
\hline 55 & $\mathrm{H}_{3} \mathrm{C}$ & $\mathrm{H}$ & $\mathrm{H}$ & 5.236 \\
\hline
\end{tabular}

Table 2-i: Molecular descriptors of (Model 1 MLR) training set used in the regression analysis

\begin{tabular}{|c|c|c|c|c|c|}
\hline Compound & T_2_0_1 & T_C_0_5 & T_2_0_2 & T_0_0_6 & Slogp \\
\hline 3 & 6 & 6 & 11 & 0 & 4.247 \\
\hline 6 & 4 & 2 & 8 & 0 & 5.19 \\
\hline 10 & 5 & 4 & 10 & 0 & 5.435 \\
\hline 13 & 6 & 5 & 11 & 0 & 3.944 \\
\hline 16 & 5 & 6 & 10 & 1 & 3.334 \\
\hline 21 & 6 & 8 & 11 & 0 & 4.301 \\
\hline 23 & 4 & 2 & 8 & 0 & 4.676 \\
\hline 26 & 4 & 2 & 8 & 0 & 4.119 \\
\hline 27 & 6 & 6 & 11 & 0 & 3.944 \\
\hline 32 & 5 & 3 & 9 & 0 & 4.547 \\
\hline 33 & 4 & 4 & 8 & 0 & 4.911 \\
\hline 36 & 6 & 7 & 9 & 2 & 4.164 \\
\hline 40 & 6 & 4 & 11 & 0 & 4.235 \\
\hline 41 & 6 & 5 & 11 & 1 & 4.247 \\
\hline 43 & 7 & 7 & 13 & 1 & 4.256 \\
\hline 44 & 6 & 4 & 11 & 0 & 3.944 \\
\hline 45 & 7 & 6 & 13 & 1 & 3.65 \\
\hline 46 & 6 & 5 & 11 & 1 & 3.944 \\
\hline 47 & 5 & 3 & 10 & 0 & 4.242 \\
\hline 49 & 7 & 6 & 13 & 0 & 3.953 \\
\hline 52 & 5 & 5 & 8 & 1 & 4.495 \\
\hline 53 & 4 & 3 & 9 & 0 & 3.877 \\
\hline 55 & 5 & 4 & 10 & 0 & 4.545 \\
\hline
\end{tabular}




\section{RESULTS}

The most remarkable 2D QSAR models employing multiple linear regression method (MLR) evaluated squared correlation coefficient value 0.8002 . The result of MLR analysis were compared with the results of Partial Least Square (PLS) regression method. T_C_0_5, T_2_0_2, s log p, T_2_0_1 and T_2_0_6 descriptors were found significant in $2 \mathrm{D}$ QSAR model.

k-Nearest Neighbour Molecular Field Analysis of best 3D QSAR model predicted $\mathrm{q}^{2}$ value 0.8790 , q2_se value 0.0794 , pred $\mathrm{r}^{2}$ value 0.9340 and pred_r ${ }^{2}$ se value 0.0540 . For predicting the inhibitory activity of methaniminium derivatives, stepwise regression method was used. The 3D model revealed that hydrophobic, electrostatic and steric descriptors exhibit a critical role in determining the inhibitory activity of this class of compounds.

\section{DISCUSSION}

\section{D-QSAR model}

Dataset of 30 compounds was taken into consideration along with their inhibitory activities. 2D-QSAR analysis was performed on the reported compounds of the training set (23) and test set (7). The model obtained thus, observed to be statistically significant. Therefore, the training set (23) and test set (7) were evaluated for the similarity of the distribution patterns of the molecules. Results indicated that the maximum of the test set is lesser than the maximum of the train set and the minimum of the test set is greater than that of the train set, which is required for further QSAR study.

The minimum 'inhibitory activity' of the test set was greater than the minimum activity of the training set and the maximum activity of the test set was less than the maximum activity of the training set. This indicated that the test set was within the activity domain of the training set. Higher mean value of test set than the training set indicated the presence of relatively more potent compounds in the test set as compared to inactive ones. Table 2-i, ii, iii and iv represent various descriptors used in training and test set of MLR and PLS regression analysis.

Best models using multiple linear regression and partial least square method

\section{Model-1 (MLR)}

$\mathrm{Ki}=0.1397( \pm 0.0209) \quad\left(\mathrm{T} \_\mathrm{C} \_0 \_5\right)+0.2246( \pm 0.0270)\left(\mathrm{T} \_2 \_0 \_2\right)+$

$0.1146( \pm 0.0440)(\mathrm{s} \log \mathrm{p})$

$-0.4670( \pm 0.0514)\left(T \_2 \_0 \_1\right)-0.0994( \pm 0.0334)\left(T \_2 \_0 \_6\right)+5.2416$

Model-2 (PLS)

$\mathbf{K i}=0.1411\left(\mathrm{~T}_{-} \mathrm{C}_{-} \mathrm{Cl} \_4\right)+-0.2032\left(\mathrm{~T}_{-} \mathrm{N}_{-} \mathrm{O} \_5\right)-0.0398$ (SsOHcount $)+$ 1.1804(chiV3Cluster)+0.0658(T_T_0_5)-0.6267(SdOcount) +4.7066

Table 2-ii: Molecular descriptors of (Model 1 MLR) test set used in the regression analysis

\begin{tabular}{lllll}
\hline Compound & T_2_0_1 & T_C_0_5 & T_2_0_2 & T_0_0_6 \\
\hline 8 & 4 & 3 & 8 & 0 \\
15 & 5 & 4 & 9 & 0 \\
18 & 5 & 5 & 9 & 0 \\
39 & 5 & 3 & 9 & 0 \\
48 & 4 & 2 & 8 & 0 \\
50 & 6 & 4 & 11 & 0 \\
51 & 6 & 4 & 11 & 0 \\
\end{tabular}

Table 2-iii: Molecular descriptors of (Model 2 PLS) training set used in the regression analysis

\begin{tabular}{|c|c|c|c|c|c|c|}
\hline Compound & T_C_Cl_4 & T_N_0_5 & SsOHcount & chiV3Cluster & T_T_0_5 & SdOcount \\
\hline 10 & 0 & 3 & 0 & 1.003 & 15 & 1 \\
\hline 13 & 1 & 2 & 0 & 0.791 & 6 & 0 \\
\hline 15 & 0 & 2 & 0 & 0.769 & 7 & 0 \\
\hline 16 & 0 & 2 & 0 & 0.67 & 8 & 0 \\
\hline 21 & 0 & 2 & 0 & 1.033 & 10 & 1 \\
\hline 23 & 0 & 2 & 1 & 1.039 & 12 & 1 \\
\hline 27 & 0 & 2 & 1 & 1.009 & 12 & 1 \\
\hline 32 & 0 & 2 & 0 & 0.665 & 6 & 0 \\
\hline 33 & 0 & 2 & 0 & 0.699 & 6 & 0 \\
\hline 39 & 0 & 3 & 0 & 1.09 & 10 & 1 \\
\hline 3 & 0 & 2 & 0 & 1.036 & 8 & 0 \\
\hline 41 & 0 & 4 & 0 & 1.067 & 11 & 1 \\
\hline 43 & 0 & 2 & 1 & 0.731 & 12 & 1 \\
\hline 45 & 0 & 5 & 0 & 1.056 & 18 & 1 \\
\hline 46 & 0 & 4 & 1 & 1.029 & 14 & 1 \\
\hline 47 & 0 & 5 & 2 & 1.066 & 17 & 1 \\
\hline 49 & 0 & 2 & 1 & 0.677 & 7 & 0 \\
\hline 50 & 0 & 2 & 0 & 0.602 & 6 & 0 \\
\hline 51 & 0 & 6 & 1 & 1.059 & 18 & 1 \\
\hline 52 & 0 & 4 & 1 & 0.991 & 12 & 1 \\
\hline 53 & 0 & 2 & 0 & 0.727 & 12 & 1 \\
\hline 55 & 0 & 2 & 0 & 0.788 & 9 & 1 \\
\hline 6 & 0 & 2 & 1 & 0.72 & 9 & 0 \\
\hline
\end{tabular}

Table 2-iv: Molecular descriptors of (Model 2 PLS) test set used in the regression analysis

\begin{tabular}{llllll}
\hline Compound & T_C_Cl_4 & T_N_0_5 & Ss0Hcount & chiV3Cluster & T_T_0_5 \\
\hline 8 & 0 & 2 & 0 & 0.67 & 8 \\
18 & 0 & 3 & 1 & 1.008 & 14 \\
26 & 0 & 3 & 0 & 1.007 & 1 \\
36 & 0 & 4 & 1 & 0.991 & 1 \\
42 & 0 & 2 & 1 & 0.785 & 1 \\
44 & 0 & 4 & 0 & 1.005 & 1 \\
48 & 0 & 4 & 1 & 1.01 & 1 \\
\end{tabular}


Table 3: Statistical parameters (uni-column statistics of model 1 MLR and model 2 PLS) for activity distribution in training and test sets

\begin{tabular}{llll}
\hline Parameters & Model 1 MLR & Model 2 PLS \\
\cline { 2 - 4 } & Training set & Test set & Training set \\
\hline Max. & 5.9200 & 5.8600 & 5.9200 \\
Min. & 5.2300 & 5.3000 & 5.2300 \\
Std. Dev. & 0.2304 & 0.2527 & 0.2364 \\
Sum & 126.2200 & 38.4900 & 126.3100 \\
Average & 5.4878 & 5.4986 & 5.4917 \\
\hline
\end{tabular}

Table 4: It shows the summary of statistical parameters for 2D-QSAR models of Amino (3-((3, 5-difluoro-4-methyl-6-phenoxypyridine-2yl) oxy) phenyl) methaniminium derivative

\begin{tabular}{lll}
\hline Statistical parameter & Best model (MLR) & Best model (PLS) \\
\hline $\mathrm{N}$ & 23 & 23 \\
Degree of freedom & 17 & 18 \\
$\mathrm{r}^{2}$ & 0.8002 & 0.7813 \\
$\mathrm{q}^{2}$ & 0.6107 & 0.5762 \\
$\mathrm{~F}$ test & 13.6181 & 16.0749 \\
$\mathrm{r}^{2}$ se & 0.1172 & 0.1222 \\
$\mathrm{q}^{2}$ se & 0.1636 & 0.1702 \\
pred_r ${ }^{2}$ & 0.5248 & 0.6621 \\
pred_r ${ }^{2}$ & 0.1744 & 0.1347 \\
\hline
\end{tabular}

MLR analysis with these newly formed datasets showed better statistically significant results (table 3 and 4 ). In the multiple regression method, T_C_0_5, T_2_0_2,s log p,T_2_0_1 and T_2_0_6 were found contributing as descriptors while in Partial Least square method,T_C_Cl_4,T_N_0_5,SsOHcount,chiV3Cluster,T_T_0_5 and SdOcount were used as descriptors. On the basis of statistical analysis, the compounds have depicted residual value (table 5) less than 1 unit.

Table 5: It represents residuals of 2 D QSAR

\begin{tabular}{|c|c|c|c|c|c|}
\hline \multirow[t]{2}{*}{ Compound No. } & \multirow[t]{2}{*}{-log (Ki)for factor Xa Actual } & \multicolumn{2}{|l|}{ Model 1 MLR } & \multicolumn{2}{|l|}{ Model 2 PLS } \\
\hline & & Predicted Ki & Residual & Predicted Ki & Residual \\
\hline 3 & 5.9208 & 5.6385 & 0.2824 & 6.0492 & 0.2093 \\
\hline 6 & 5.8861 & 5.6468 & 0.2393 & 5.7021 & 0.0004 \\
\hline 8 & 5.8697 & 5.7126 & 0.1571 & 5.6171 & 0.0718 \\
\hline 10 & 5.8500 & 5.7376 & 0.1124 & 5.6407 & 0.1229 \\
\hline 13 & 5.7696 & 5.4640 & 0.3055 & 5.7696 & 0.1490 \\
\hline 15 & 5.7447 & 5.6057 & 0.1390 & 5.6682 & 0.1195 \\
\hline 16 & 5.7447 & 5.6768 & 0.0679 & 5.6171 & 0.0108 \\
\hline 18 & 5.6990 & 5.5788 & 0.1202 & 5.5410 & 0.1619 \\
\hline 21 & 5.6778 & 5.7252 & -0.0474 & 5.5505 & 0.0062 \\
\hline 23 & 5.6576 & 5.5879 & 0.0697 & 5.6492 & 0.0103 \\
\hline 26 & 5.6198 & 5.5240 & 0.0958 & 5.4481 & -0.0399 \\
\hline 27 & 5.6198 & 5.5043 & 0.1155 & 5.6138 & 0.0223 \\
\hline 32 & 5.4949 & 5.3710 & 0.1239 & 5.4797 & -0.1146 \\
\hline 33 & 5.4815 & 5.8942 & -0.4128 & 5.5199 & -0.1292 \\
\hline 36 & 5.3468 & 5.2201 & 0.1267 & 5.3177 & 0.0164 \\
\hline 39 & 5.3010 & 5.2996 & 0.0015 & 5.4146 & 0.0500 \\
\hline 40 & 5.3010 & 5.3576 & -0.0566 & 5.2836 & 0.0144 \\
\hline 41 & 5.3010 & 5.2999 & 0.0012 & 5.2500 & -0.0084 \\
\hline 43 & 5.3010 & 5.3636 & -0.0626 & 5.2856 & -0.1942 \\
\hline 44 & 5.3010 & 5.2249 & 0.0762 & 5.3084 & -0.0626 \\
\hline 45 & 5.3010 & 5.1545 & 0.1466 & 5.4942 & -0.0605 \\
\hline 46 & 5.3010 & 5.2651 & 0.0359 & 5.3626 & 0.0256 \\
\hline 47 & 5.3010 & 5.4611 & -0.1601 & 5.3605 & -0.2198 \\
\hline 48 & 5.3010 & 5.5719 & -0.2709 & 5.2744 & -0.1054 \\
\hline 49 & 5.3010 & 5.2886 & 0.0124 & 5.5198 & 0.0453 \\
\hline 50 & 5.3010 & 5.2249 & 0.0762 & 5.4054 & 0.1138 \\
\hline 51 & 5.3010 & 5.4125 & -0.1115 & 5.2547 & -0.0608 \\
\hline 52 & 5.3010 & 5.3205 & -0.0194 & 5.1862 & 0.0345 \\
\hline 53 & 5.2840 & 5.7611 & -0.4771 & 5.3208 & 0.1779 \\
\hline 55 & 5.2366 & 5.6356 & -0.3990 & 5.1955 & 0.2429 \\
\hline
\end{tabular}




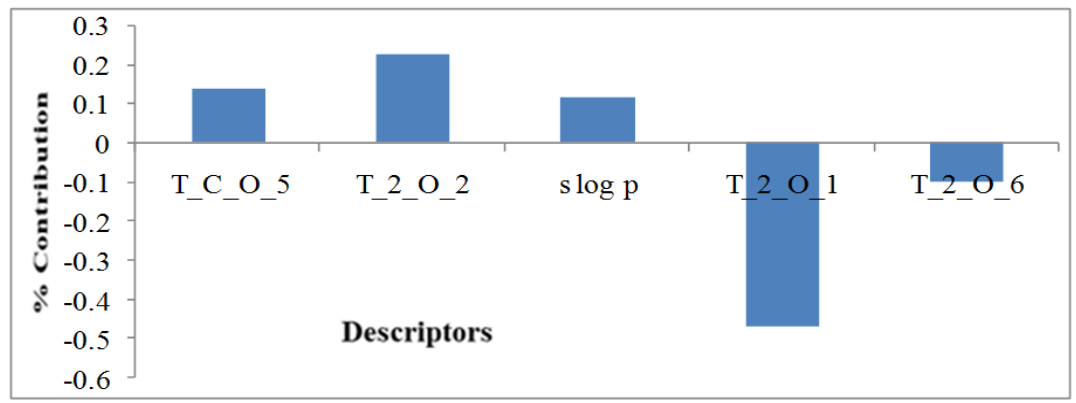

Fig. 1: It shows percentage contribution of descriptors of 2D QSAR (model 1 MLR)

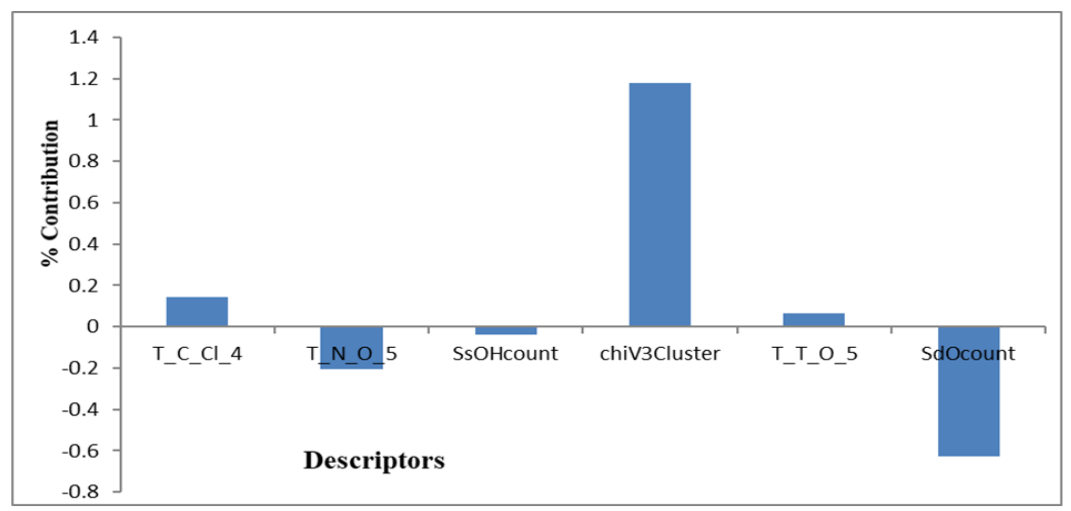

Fig. 2: It shows percentage contribution of descriptors of 2D QSAR (model 2 PLS)

The 'fitness plot' which is a plot of experimental activities with predicted activity of test set compounds and training set compounds for each model shows that built models are statistically significant. This provides an idea about the fitness of the model and also about the predictive ability of external test set (fig. 3 and fig. 4).

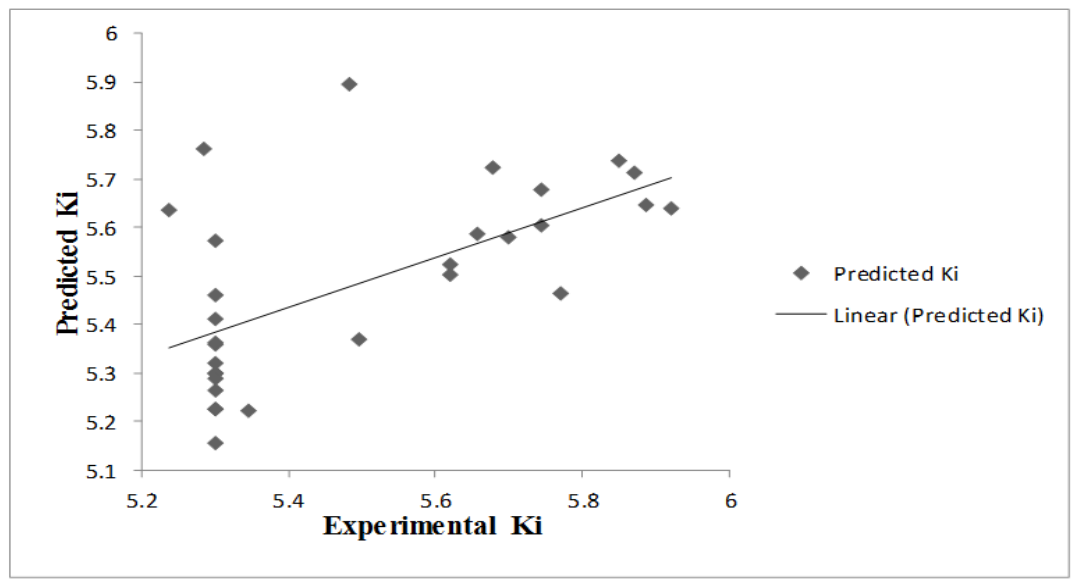

Fig. 3: It shows experimental vs. predicted activities for training and test set molecules from the best predictive MLR model (model 1)

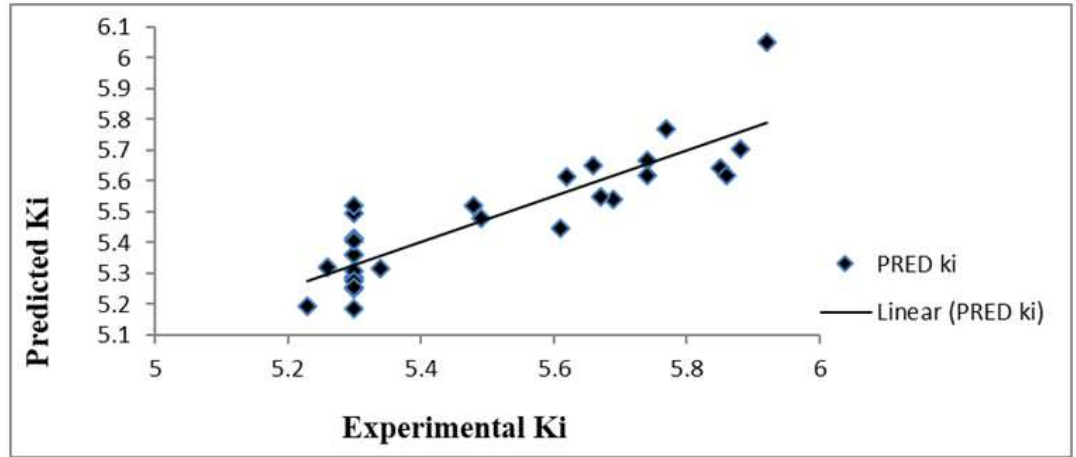

Fig. 4: It represents experimental vs. predicted activities for training and test set molecules from the best predictive PLS model (model 2) 
In the generated model of QSAR by MLR method, physicochemical descriptor slogp and alignment independent (AI) descriptors T_C_O_5 and T_2_O_2 contributed positively, therefore, molecules showing higher values of T_C_O_5, T_2_O_2 and slogp will have good inhibitory activity.

The model indicates the negative contribution of alignment independent topological descriptors T_2_O_1 and T_2_O_6 thus molecules showing higher values of T_2_0_1 and T_2_0_6 will show reduced inhibitory activity. Physicochemical descriptor slogp signifies log of the octanol/water partition coefficient. Molecules with high partition coefficient tends to remain in the lipid bilayer that is in the hydrophobic phase. As log p increases, the probability of molecule reaching to its critical binding site increases, therefore, crossing of lipid bilayer increases. Another alignment independent topological descriptor T_2_0_1 and T_2_O_6 contributed negatively to the model developed by MLR method and is inversely proportional to the activity. T_2_0_1 and T_2_0_6 which contributed negatively represent the count of the number of double-bonded atoms separated from oxygen atom by one bond distance and six bond distances respectively in a molecule. The negative contribution of these two descriptors suggests that lower values results in good inhibitory activities while greater values of T_2_0_1 and T_2_0_6 results in reduced inhibitory activities. 2D-QSAR study reveals that the physicochemical descriptor plays a pivotal role and is the key descriptor.

In the model generated by PLS method, Alignment Independent (AI) descriptors T_C_Cl_4 and T_T_O_5 and chiV3Cluster exhibit positive contribution while T_N_O_5 and SsOHcount shows negative contribution. chiV3Cluster signifies atomic valence connectivity index (order 3) [Hall 1991]. Molecules with higher values of T_C_Cl_4 and T_T_0_5 and chiV3Cluster will show good inhibitory activity and molecules with higher values of T_N_O_5 and SsOHcount will exhibit reduced inhibitory activity. These descriptors (Alignment Independent) can be calculated as discussed in Baumann's paper [27]. This descriptor specifies the total number of oxygen atoms linked with one single bond. The descriptor SdOcount denotes the total number of hydroxyl group linked with one single bond.

The most significant 2D models of methaniminium derivatives using MLR and PLS method was compared and it was concluded that MLR method generated the better model. The multiple linear regression (MLR) analysis calculated squared correlation coefficient $\mathrm{r}^{2}$ value 0.8002 , predictive squared correlation coefficient $\mathrm{q}^{2}$ value $0.6107, \mathrm{~F}$ test value 13.6181, root mean square error $r^{2}$ sevalue 0.1172, predictive squared correlation coefficient standard error $\mathrm{q}^{2}$ se value 0.1636 , pred_ $\mathrm{r}^{2}$ value 0.5248 and root mean square error predicted (pred_r ${ }^{2}$ se) value 0.1744 .

\section{D-QSAR modeling}

To perform 3D QSAR, the dataset of 30 molecules were taken and it was divided into training ( 22 compounds) and test set ( 8 compounds). In this, Ki values act as dependent variable while all calculated 3D descriptors act as independent variables.

\section{Molecular modeling and alignment of molecules}

Conformational search method (grid search) was performed which gives all potential conformations, by changing consistently each torsion angle of the molecule by increasing it gradually but at the same time maintaining the bond length and bond angles. The conformers with the lowest energy (therefore more stable) were chosen. Template-based alignment method was applied to align all the molecules of the series. For this purpose, the most active compound with minimum energy conformation was used as the template and all the compounds were aligned on that [28].

\section{Computation of field descriptors}

In order to perform 3D QSAR, steric and electrostatic field descriptors were computed having cutoffs of $30.0 \mathrm{kcal} / \mathrm{mol}$ and 10.0 $\mathrm{kcal} / \mathrm{mol}$ respectively. The Gasteiger-Marsili [29] charge type was chosen. Distance-dependent dielectric function was selected; keeping the value of dielectric constant 1.0. A carbon atom with charge 1.0 was set as the probe. Using this, electrostatic (1040 descriptors), steric (1040 descriptors) and hydrophobic descriptors (1040 descriptors) were calculated and altogether 3120 descriptors were obtained. From this set of descriptors, invariable columns were removed as they have no contribution towards QSAR.

\section{K-nearest neighbour molecular field analysis (kNN-MFA)}

The foremost need of kNN-MFA is an alignment of molecules. After that, a rectangular grid is generated all around the molecules. Then at the lattice points of the grid, electrostatic and steric energies are calculated which are further employed for relationship generation with the help of kNN method to determine distances between molecules [30].

\section{k-NN-MFA with stepwise forward-backward (SW-FB) variable selection method}

Forward and backward stepwise selection method was employed to generate k-NN-MFA models with cross-correlation limit fixed to 1.0 and $\mathrm{q}^{2}$ was taken as term selection criteria. The values which were set for F-test "in" was 4.0 and F-test 'out' as 3.99. Variance cut off was kept at $0.0 \mathrm{kcal} / \mathrm{mol} \mathrm{A}^{\circ}$ and scaling was set as Auto Scaling. For k-Nearest Neighbour parameter setting, the number of maximum neighbors were set to 5 , numbers of minimum neighbors were set to 2 and distance based weighted average was chosen as prediction method.

\section{D QSAR model generation and interpretation}

K-NN-MFA method was employed for the generation of 3D-QSAR models. For this purpose, the dataset of 30 compounds was used which was further divided into training and test set. The k-NN-MFA models (3-4) were obtained utilizing a training set of 22 compounds and test set of 8 compounds. The hydrophobicity $(\mathrm{H})$, electrostatic (E) and steric (S) descriptors particularize the regions, whereby changing the structure of the compound of the training set; the activities of the compound may increase or decrease. The descriptors are associated with numbers which correspond to its place in the 3D MFA grid. Many statistically significant models were generated using stepwise forward, backward variable selection method, from which model 4 meets the selection criterion at its best. The criterion of selection of the best model was internal and external predictive ability of the model. This was achieved by $\mathrm{q}^{2}$ which represents the internal predictive ability of the model and by pred_r ${ }^{2}$, which anticipate the activity of an external test set.

Table 6-i: It shows 3D molecular descriptors of training set used in the k-NN-MFA (model 4)

\begin{tabular}{|c|c|c|c|c|}
\hline Compound & E_513 & S_434 & E_1897 & H_1619 \\
\hline 10 & -0.05751 & -0.00737 & 0.261705 & 0.362097 \\
\hline 16 & -0.07086 & -0.02495 & 0.214728 & 0.420844 \\
\hline 18 & 0.052579 & -0.04344 & 0.176186 & 0.345001 \\
\hline 23 & -0.05253 & -0.08391 & 0.187079 & 0.288696 \\
\hline 26 & -0.11056 & 30 & 0.116523 & 0.421608 \\
\hline 27 & 0.056585 & -0.03845 & 0.075065 & 0.386805 \\
\hline 32 & -0.00559 & -0.01633 & 0.195233 & 0.402994 \\
\hline 40 & -0.4109 & -0.02305 & 0.188377 & 0.358622 \\
\hline 41 & 0.038644 & -0.11408 & 0.068592 & 0.327683 \\
\hline 42 & 0.210907 & -0.02251 & 0.172865 & 0.383975 \\
\hline 43 & -0.00752 & -0.03611 & 0.142588 & 0.369393 \\
\hline 44 & -0.15747 & -0.04573 & 0.082705 & 0.342664 \\
\hline
\end{tabular}




\begin{tabular}{|c|c|c|c|c|}
\hline 45 & 0.143883 & -0.04939 & 0.140757 & 0.362183 \\
\hline 47 & 0.367139 & -0.13843 & -0.14734 & 0.313056 \\
\hline 48 & 0.025765 & -0.10398 & -0.06093 & 0.322267 \\
\hline 50 & -0.00545 & -0.012 & 0.082555 & 0.384552 \\
\hline 51 & 0.182347 & -0.02065 & 0.129918 & 0.316739 \\
\hline 52 & -0.12303 & -0.0318 & 0.081317 & 0.349608 \\
\hline 53 & 0.016184 & -0.0914 & 0.079591 & 0.371672 \\
\hline 55 & -0.04311 & -0.11282 & 0.095685 & 0.359617 \\
\hline 6 & -0.06325 & -0.02067 & 0.1859 & 0.313061 \\
\hline 8 & 0.066054 & -0.02051 & 0.124146 & 0.397901 \\
\hline
\end{tabular}

Table 6-ii: It represents 3D molecular descriptors of test set used in the k-NN-MFA (model 4)

\begin{tabular}{llll}
\hline Compound & E_513 & S_434 & E_1897 \\
\hline 13 & 0.027768 & -0.0115 & 0.099411 \\
15 & 0.045428 & -0.04021 & 0.19768 \\
21 & -0.16166 & -0.04177 & 0.286382 \\
33 & 0.191621 & -0.02135 & 0.159468 \\
36 & 0.359089 & -0.06424 & 0.027854 \\
39 & 0.166087 & -0.09394 & 0.161573 \\
46 & 0.170558 & -0.02543 & 0.07286 \\
49 & 0.026955 & -0.01761 & 0.451448 \\
\hline
\end{tabular}

Analysis of statistical parameters suggests that the maximum of test set compound of Amino (3-( (3, 5-difluoro-4-methyl-6phenoxypyridine-2-yl) oxy) phenyl) methaniminium derivative is less than the maximum of the training set of compounds. The minimum binding affinity of training set compounds is also less than the minimum of test set compounds. The average of test set compounds is higher than the average of train set compounds which denotes that comparatively more active compounds are present in test set than in train set. The statistical parameters are shown in table 8.

Table 7: It shows statistical parameters (uni-column statistics) for biological activity distribution in training and test sets of 3D QSAR (model 4 SW-FB)

\begin{tabular}{lll}
\hline Parameters & Training set & Test set \\
\hline Max. & 5.8900 & 5.7700 \\
Min. & 5.2300 & 5.300 \\
Std. Dev. & 0.2283 & 0.2087 \\
Sum & 120.24 & 43.9200 \\
Average & 5.465 & 5.4900 \\
\hline
\end{tabular}

\section{Models using k-NN-MFA method}

\section{Model-3 (SW-FB)}

$\mathrm{Ki}=$ E_513 (-0.0709,-0.0525) E_2066 (0.0755, 0.0914) E_1359 ($10.0000,-10.0000)$

\section{Model-4 (SW-FB)}

$\mathrm{Ki}=\mathrm{E} \_513(-0.0575,-0.0525) \quad$ S_434 $(-0.0839,-0.0074) \quad$ E_1897 $(0.1871,0.2617)$ H_1619 $(0.2887,0.3621)$

The model 4, which is obtained by SW-FB selection method, is chosen as the best model based on an internal prediction. The leave one out cross-validation squared correlation coefficient, $\mathrm{q}^{2}$ was calculated as 0.8790 which indicated good prediction. It also shows better prognostic power for the external test set having predictive squared correlation coefficient value (predicted $r^{2}$ ) equal to 0.9340 which is equal to $93 \%$ predictive power. Table 3.10 depicts actual and predicted values of test set and training set data by model 4 with their residual values.

The best 3D-QSAR model (model 4) established that electrostatic, steric and hydrophobic interactions contribute majorly in prediction. In this, E_513 and E_1897 are electrostatic descriptors while S_434 is steric descriptor. H_1619 is a hydrophobic descriptor.

The negative value of the electrostatic field descriptor shows that negative electronic potential is needed to enhance the activity, therefore more electronegative groups are favoured in that position. Likewise, negative values of steric descriptors suggest that negative values of steric potential are preferable for activity and less bulkier groups as substitutes are favoured in that particular area. Steric descriptor with positive range denotes that more bulky substituents are favoured in that area.

Table 8: It exhibits summary of statistical parameters for 3D-QSAR models (model 3 and 4) of Amino (3-((3, 5-difluoro-4-methyl-6phenoxypyridine-2-yl) oxy) phenyl) methaniminium derivative using similar test set and training set

\begin{tabular}{|c|c|c|}
\hline Statistical parameter & SW-FB selection model 3 & SW-FB selection model 4 \\
\hline k Nearest Neighbour & 2 & 2 \\
\hline $\mathrm{n}$ & 22 & 22 \\
\hline Degree of freedom & 18 & 17 \\
\hline$q^{2}$ & 0.8475 & 0.8790 \\
\hline $\mathrm{q}^{2} \_\mathrm{se}$ & 0.0886 & 0.0794 \\
\hline Predr ${ }^{2}$ & 0.4965 & 0.9340 \\
\hline pred_r ${ }^{2}$ se & 0.1525 & 0.0540 \\
\hline
\end{tabular}




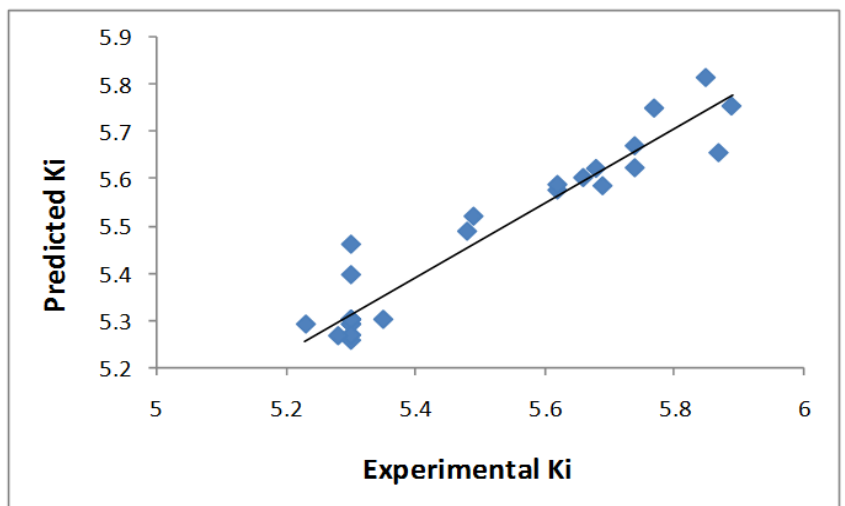

Fig. 5: It shows the graph of experimental versus predicted Ki using model-4 (3D-QSAR)

Table 9: It represents residuals of experimental and predicted inhibitory activities of 3D QSAR for models 3 and 4 containing a test set and training set data

\begin{tabular}{|c|c|c|c|c|c|}
\hline \multirow[t]{2}{*}{ Compound No. } & \multirow{2}{*}{$\begin{array}{l}\text {-Log (Ki) for factor } \mathrm{Xa} \\
\text { experimental }\end{array}$} & \multicolumn{2}{|l|}{ Model 3} & \multicolumn{2}{|l|}{ Model 4} \\
\hline & & predicted $\mathrm{Ki}$ & Residual & Predicted Ki & Residual \\
\hline 10 & 5.85 & 5.81448 & 0.03552 & 5.81451 & 0.03549 \\
\hline 13 & 5.77 & 5.58628 & 0.18372 & 5.74955 & 0.02045 \\
\hline 15 & 5.74 & 5.59046 & 0.14954 & 5.62259 & 0.11741 \\
\hline 16 & 5.74 & 5.77543 & -0.03543 & 5.66933 & 0.07067 \\
\hline 18 & 5.69 & 5.68094 & 0.00906 & 5.58423 & 0.10577 \\
\hline 21 & 5.68 & 5.79688 & -0.11688 & 5.62101 & 0.05899 \\
\hline 23 & 5.66 & 5.81551 & -0.15551 & 5.60157 & 0.05843 \\
\hline 26 & 5.62 & 5.59013 & 0.02987 & 5.575 & 0.045 \\
\hline 27 & 5.62 & 5.57641 & 0.04359 & 5.58694 & 0.03306 \\
\hline 32 & 5.49 & 5.47969 & 0.01031 & 5.51941 & -0.02941 \\
\hline 33 & 5.48 & 5.48031 & -0.00031 & 5.48747 & -0.00747 \\
\hline 36 & 5.35 & 5.3 & 0.05 & 5.3 & 0.05 \\
\hline 39 & 5.3 & 5.48592 & -0.18592 & 5.3 & 0 \\
\hline 40 & 5.3 & 5.45945 & -0.15945 & 5.3 & 0 \\
\hline 41 & 5.3 & 5.44814 & -0.14814 & 5.25546 & 0.04454 \\
\hline 42 & 5.3 & 5.49065 & -0.19065 & 5.3 & 0 \\
\hline 43 & 5.3 & 5.26515 & 0.03485 & 5.39504 & -0.09504 \\
\hline 44 & 5.3 & 5.3284 & -0.0284 & 5.26669 & 0.03331 \\
\hline 45 & 5.3 & 5.3 & 0 & 5.3 & 0 \\
\hline 46 & 5.3 & 5.3 & 0 & 5.29025 & 0.00975 \\
\hline 47 & 5.3 & 5.3 & 0 & 5.3 & 0 \\
\hline 48 & 5.3 & 5.31608 & -0.01608 & 5.2901 & 0.0099 \\
\hline 49 & 5.3 & 5.28986 & 0.01014 & 5.28978 & 0.01022 \\
\hline 50 & 5.3 & 5.28997 & 0.01003 & 5.45989 & -0.15989 \\
\hline 51 & 5.3 & 5.3 & 0 & 5.3 & 0 \\
\hline 52 & 5.3 & 5.43031 & -0.13031 & 5.26635 & 0.03365 \\
\hline 53 & 5.28 & 5.3 & -0.02 & 5.26519 & 0.01481 \\
\hline 55 & 5.23 & 5.3 & -0.07 & 5.28987 & -0.05987 \\
\hline 6 & 5.89 & 5.69988 & 0.19012 & 5.75392 & 0.13608 \\
\hline 8 & 5.87 & 5.65471 & 0.21529 & 5.65457 & 0.21543 \\
\hline
\end{tabular}

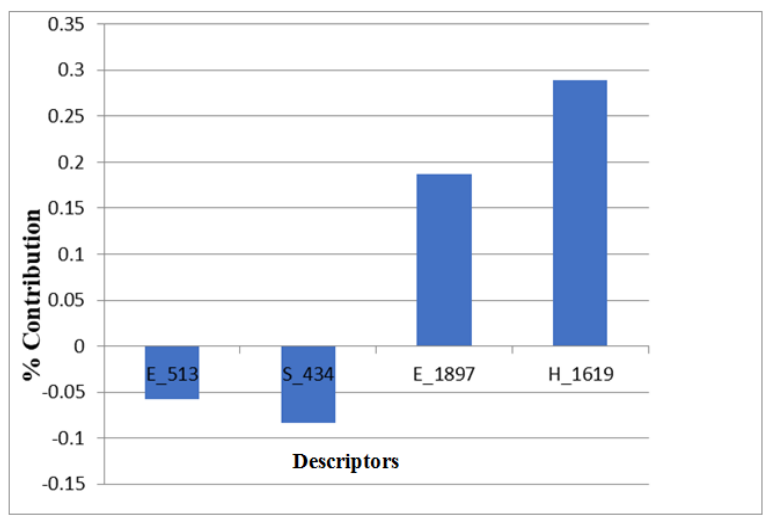

Fig. 6: It shows a contribution chart of the descriptors of 3D-QSAR model (model 4) 


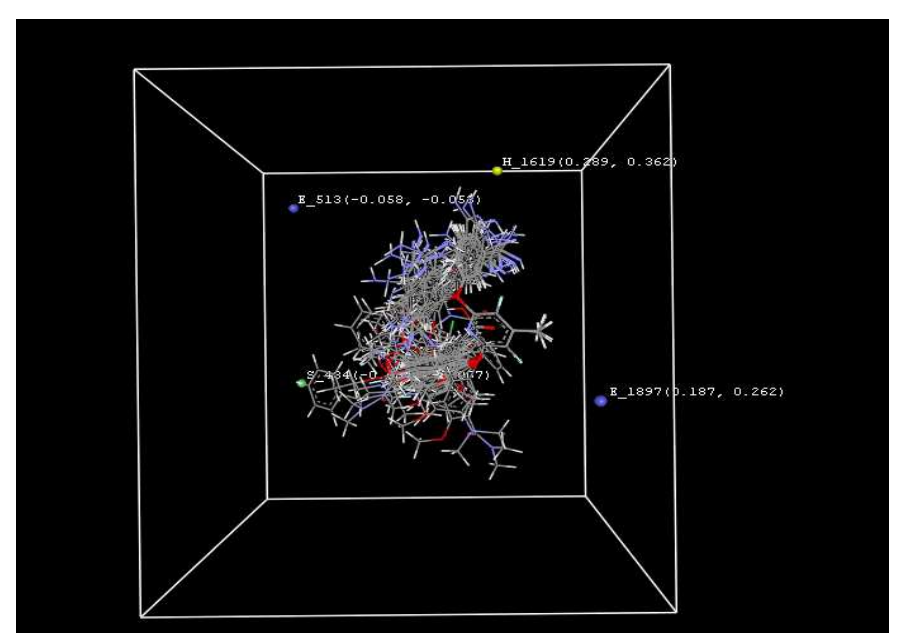

Fig. 7-i: It represents contour plots of 3D-QSAR (model 4) with important hydrophobic, electrostatic and steric fields

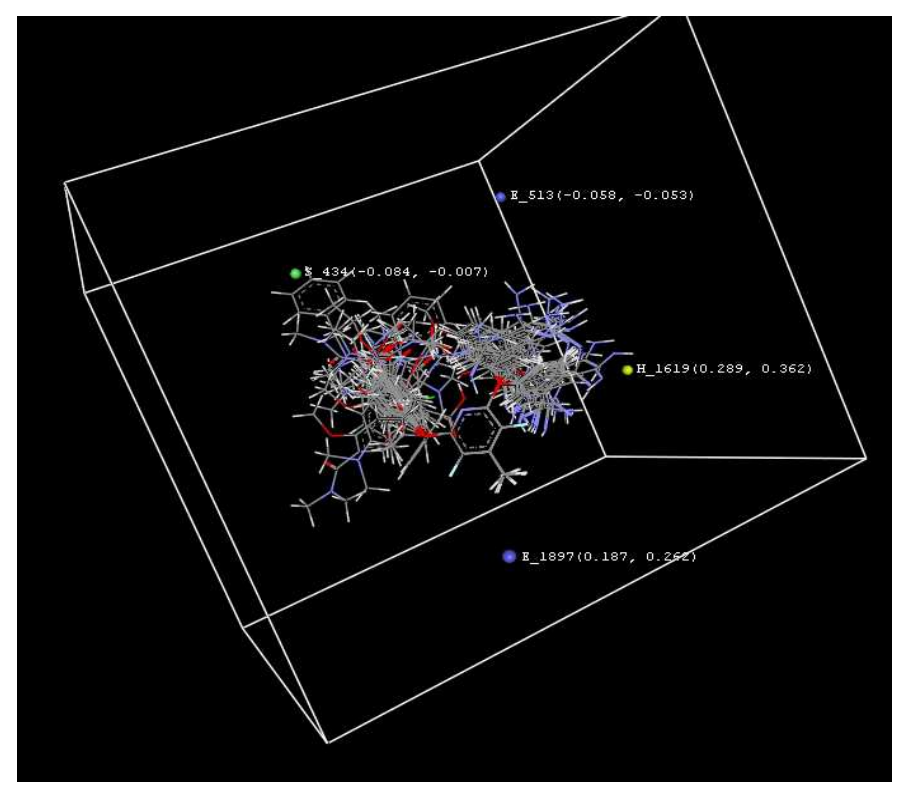

Fig. 7-ii: It represents contour plots of 3D-QSAR (model 4) with important hydrophobic, electrostatic and steric fields

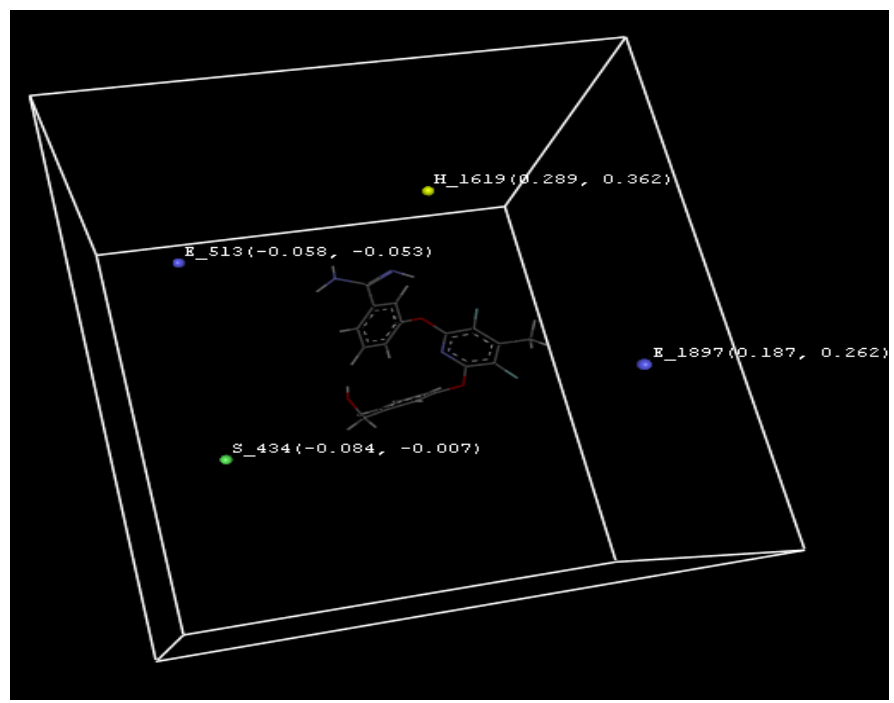

Fig. 8: It shows 3D structure of most active molecule 6 


\section{CONCLUSION}

On the basis of the best model of 3D QSAR (model 4), the following outcomes were observed for designing of new molecules with regard to electrostatic, steric and hydrophobic fields. The positive value of electrostatic field E_1897 (0.1871, 0.2617) denotes that positive electrostatic potential is preferable to enhance the inhibitory activity of compound and therefore substituents with lesser electronegativity values are favourable at that particular place while negative value of electrostatic field E_513 (-0.0575,-0.0525) shows that for enhancing the activity of compound, the substituent groups with higher electronegativity values are found to be suitable for that particular region. The steric field S_434 (-0.0839,-0.0074) contributed negatively, which suggests that less bulky substituent groups are preferable in that domain. The positive hydrophobic field descriptors H_1619 (0.2887, 0.3621) indicates that hydrophilic groups in that region can raise the activity of the compounds.

Thus, contour plots of KNN-MFA method furnish additional information on the relationship between the structure of compounds and their inhibitory activities which can be employed to construct newer factor Xa inhibitors.

\section{AUTHORS CONTRIBUTIONS}

Dr. Smita Suhane is responsible for QSAR analysis and drafting the manuscript.

Dr. A. G. Nerkar has provided advice and guidance during the work.

Dr. Kumud Modi has provided guidance.

Dr. Sanjay D. Sawant has provided guidance during the work.

\section{CONFLICTS OF INTERESTS}

There are no conflicts of interest by any of the authors

\section{REFERENCES}

1. Johnson M, Maggiora GM. Concepts and applications of molecular similarity. Wiley; 1990.

2. Hansch, Corwin. Exploring QSAR. Washington DC. American Chemical Society; 1995.

3. Hansch, Corwin. The expanding role of quantitative structureactivity relationships (QSAR) in toxicology. Toxicol Lett 1995; 79:45-53

4. Hansch, Corwin. Antitumor 1-(X-aryl)-3, 3-dialkyltriazenes. 2. on the role of correlation analysis in decision making in drug modification. Toxicity quantitative structure-activity relationships of 1-(X-phenyl)-3, 3-dialkyltriazenes in mice.J Med Chem 1978;21:574-7.

5. Bradbury SP. Predicting modes of toxic action from chemical structure: an overview. SAR and QSAR in Environ Res 1994;2:89-104.

6. Russom CL, SP Bradbury, AR Carlson. Use of knowledge bases and QSARs to estimate the relative ecological risk of agrichemicals: a problem formulation exercise. SAR and QSAR in Environ Res 1995;4:83-95.

7. Schultz, Terry W, JR Seward. Health-effects related structuretoxicity relationships: a paradigm for the first decade of the new millennium. Sci Total Environ 2000;249:73-84.

8. Shi Leming M. QSAR models using a large diverse set of estrogens. J Chem Inf Comput Sci 2001;41:186-95.
9. Tong W. An integrated computational approach for prioritizing potential estrogenic endocrine disruptors. Proceedings of the International Symposium on Environmental Endocrine Disruptors; 1999.

10. Wen, Yuan Hua, Jacob Kalff, Robert Henry Peters. Pharmacokinetic modeling in toxicology: a critical perspective. Environ Rev 1999;7:1-18.

11. Furie B, Furie BC. Mechanisms of thrombus formation. New England J Med 2008;359:938-49.

12. Handin Rl, Kasper DL, Braunwald E, Fauci AS. Harrison's principles of internal medicine. 16th ed. New York, NY. McGraw-Hill Medical Publishing Division; 2005. p. 337-43.

13. Waldo AL. Anticoagulation: stroke prevention in patients with atrial fibrillation. Med Clin North Am 2008;92:143-59.

14. Fuster V, Moreno PR, Fayad ZA. Atherothrombosis and highrisk plaque: part I: evolving concepts. J Am Coll Cardiol 2005;46:937-54.

15. Tapson VF. Acute pulmonary embolism. N Engl J Med 2008;1037-52. http://dx.doi.org/10.1136/heart.85.2.229

16. Colman RW, VJ Marder, AW Clowes. Overview of coagulation, fibrinolysis, and their regulation. Hemostasis and Thrombosis: Basic Principles and Clinical Practice Philadelphia; 2006. p. 17-20.

17. Hyers TM. Management of venous thromboembolism: past, present, and future. Arch Intern Med 2003;163:759-68.

18. Hirsh J, O'donnell M, Weitz JI. New anticoagulants Blood; 2005. p. 105-453.

19. Weitz JI, Hirsh J, Samama MM. New antithrombotic drugs: American college of chest physicians evidence-based clinical practice guidelines. 8th ed. Chest J 2008;133:234S-56S.

20. Turpie AG. New oral anticoagulants in atrial fibrillation. Eur Heart J 2008;29:155-65.

21. Leung LL, Mannucci PM, Landaw SA. Anticoagulants other than heparin and warfarin. Pharmas Guide Hematology; 2014.

22. Becker RC. Next-generation antithrombin therapies. J Invasive Cardiol 2009;21:179-85.

23. Franchini M, Mannucci PM. A new era for anticoagulants. Eur ] Intern Med 2009;365:562-68.

24. Böhm M, Sturzebecher J, Klebe G. Three-dimensional quantitative structure-activity relationship analyses using comparative molecular field analysis and comparative molecular similarity indices analysis to elucidate selectivity differences of inhibitors binding to trypsin, thrombin, and factor Xa. J Med Chem 1999;42:458-77.

25. Phillips, Gary. Design, synthesis, and activity of a novel series of factor Xa inhibitors: optimization of arylamidine groups 1, 2. J Med Chem 2002;45:2484-93.

26. VLife MDS. version 4.3, VLife Sciences Technologies Pvt. Ltd. Pune India; 2008.

27. K Baumann. An alignment-independent versatile structure descriptor for QSAR and QSPR based on the distribution of molecular features. J Chem Inf Comput Sci 2002;42:26-35.

28. Balajee R, Dhanarajan MS. 3D QSAR studies of identified compounds as potential inhibitors for anti-hyperglycemic targets. Asian J Pharm Clin Res 2015;8:362-4.

29. Gasteiger J, Marsili M. Iterative partial equalization of orbital electronegativity-a rapid access to atomic charges. Tetrahedron 1980;36:3219-28.

30. Shen Min. Quantitative structure-activity relationship analysis of functionalized amino acid anticonvulsant agents using $\mathrm{k}$ nearest neighbour and simulated annealing PLS methods. J Med Chem 2002;45:2811-23. 